DOI: $10.12731 / 2306-1561-2013-4-36$

\title{
PERFORMANCE EVALUATION IN DYNAMIC ROUTING PROTOCOL STREAMING VIDEO
}

\author{
Antonova A.A.
}

\section{Abstract}

The simulation results and comparative performance analysis of routing protocols EIGRP and OSPF, within streaming video in real time. Developed a simulation tool for the design and modeling of local and global networks, computer systems, applications and distributed systems, OPNET. Network models were created based on the routing protocols EIGRP and OSPF. Evaluation of these routing protocols has been done on the basis of quantitative indicators such as the convergence time delay variation of packets through the delay and bandwidth. Two scenarios were considered network consisting of six interconnected subnetworks, where routers subnets configured to work with EIGRP and OSPF routing protocols. The results indicate that the routing protocol EIGRP, streaming video applications in real time, is more efficient than the routing protocol OSPF.

Keywords: EIGRP, OSPF, routing protocols, modeling, network topology.

\section{УДК 004.4}

\section{ОЦЕНКА ЭФФЕКТИВНОСТИ ПРОТОКОЛОВ ДИНАМИЧЕСКОЙ МАРШРУТИЗАЦИИ ПРИ ПЕРЕДАЧЕ ПОТОКОВОГО ВИДЕО}

\section{Антонова A.A.}

\begin{abstract}
Аннотация
Представлены результаты моделирования $u$ сравнительного анализа производительности протоколов мармрутизации EIGRP и OSPF, в рамках передачи потокового видео в формате реального времени. Для моделирования использовалась средство для проектирования и моделирования локальных и глобальных сетей, компьютерных систем, приложений и распределенных систем, ОРNET. Были созданы сетевые модели на основе протоколов мармрутизации EIGRP и OSPF. Оценка этих протоколов маршрутизачии была сделана на основе количественных показателей, таких как, время сходимости, изменение задержки пакетов, сквозные задержки и пропускная способность. Были рассмотрены два сиенария сети, состоящих из шести взаимосвязанных подсетей, где маршрутизаторы подсетей настроены на работу с EIGRP и OSPF протоколами маршрутизачии. Результаты показывают, что протокол мармрутизачии EIGRP, в приложениях потокового видео реального времени, работает эффективнее, чем протокол маршрутизаџии OSPF.
\end{abstract}


Ключевые слова: EIGRP, OSPF, марирутизация, протоколь, моделирование, топология сети.

\section{Введение}

В настоящее время существует большое количество исследований, построенных на сравнительном анализе протоколов маршрутизации EIGRP и OSPF. Однако малое количество исследований направлено на предмет эффективности их применения, относительно сетевых приложений передачи потокового видео в режиме реального времени. Сопоставление и оценка протоколов маршрутизации рассматривались на основании параметров, таких как: конвергенция сетей, конвергенция деятельности, загрузка процессора, пропускная способность, задержка очереди, загруженность полосы пропускания и т.д.

\section{Сетевая конфигурация}

Были рассмотрены два сценария работы сети, состоящих из шести взаимосвязанных подсетей, где маршрутизаторы подсетей настроены на работу с EIGRP и OSPF протоколами маршрутизации. Каждая подсеть состоит из маршрутизаторов, коммутаторов, рабочих станций и т.д. Конфигурационные возможности OPNET позволяют генерировать трафик различных приложений, в том числе и трафик потокового видео, выбранный для моделирования и анализа $[1,5,6]$. Для использования дополнительной конвергенции сети, каналы отказа были первично настроены. Видео сервер подключен к сети передающей потоковое видео. Сервисы качества обслуживания (QoS) играют важную роль для обеспечения наилучшего качества и гарантии минимальных заторов в сети. Для реализации QoS, используется метод WFQ (Weighted Fair Queuing), предоставляющий механизм планирования пакетных потоков данных с различными приоритетами, который позволяет задать различные приоритеты обработки пакетов на основе дифференцированного обслуживания.

Маршрутизаторы соединены между собой PPP_DS1 дуплексным каналом связи. Коммутаторы связанны с маршрутизаторами каналами связи аналогичного качества. Рабочие станции подключены к коммутаторам 10BaseT дуплексными каналами. Bce маршрутизаторы сети настроены на работу с EIGRP. Индивидуальная DES статистика выбрана для определения показателей эффективности и оценки поведения EIGRP. Установлено, что время выполнения моделирования 900 секунд. После этого, настраивается OSPF аналогичным способом.

\section{Исследование производительности системы}

После разработки модели исследовались показатели производительности системы: продолжительность конвергенции, задержка пакетов (так же известна как Jitter), сквозное качество обслуживания (end-to-end), метрики пропускной способности (используются для определения эксплуатационного качества протокола). 
Продолжительность конвергенции показывает, насколько быстро маршрутизаторы сети достигают состояния сходимости. При использовании протокола маршрутизации, маршрутизаторы обмениваются друг с другом информацией о топологии сети. При изменении топологии сети, маршрутизаторы обмениваются известной топологией, пока информация об изменениях не распространятся по всей сети. Jitter определяется какая задержка при получении пакетов [2]. Передающая сторона посылает пакеты непрерывно и с установленным интервалом друг от друга. В связи с перегрузкой сети, не правильной обработкой пакетов и системных сбоев, устойчивость потока нарушается. Этот фактор резко снижает скорость и качество видео потока.

Сквозное качество обслуживания (end-to-end) описывает время, затраченное на доставку пакета от источника до места назначения. End-to-end задержки имеют решающее значение при передаче информации в режиме реального времени. Если пакеты прибывают слишком поздно, информация теряется, что в свою очередь негативно влияет на качество полученной информации, в нашем случае, сильно ухудшится качество потокового видео.

Пропускная способность является ключевым параметром для определения скорости, с которой пакеты будут успешно доставлены через каналы связи. Пропускная способность обычно измеряется в bits/s или bps, иногда в пакетах данных за секунду или просто пакетах [3].

Для выявления изменения скорости передачи данных, в двух способах передачи данных, для PPP_DS1 канала $1.544 \mathrm{Mbps}$, рассматривалась фоновая загруженность каждой сети, изменяя загруженность канала и анализируем изменение определенных параметров. Загруженность канала установлена и увеличивается от $0 \%$ до 80\%, как показано в таблице 1.

Таблица 1. Значения загруженности канала.

\begin{tabular}{|c|c|c|}
\hline Время (сек.) & Загруженность канала (\%) & Значение канала (bps) \\
\hline 0 & 0 & 0 \\
\hline 200 & 20 & $308800(1544000 * 0.2)$ \\
\hline 400 & 40 & $617600(1544000 * 0.4)$ \\
\hline 600 & 60 & $926400(1544000 * 0.6)$ \\
\hline 800 & 80 & $1235200(1544000 * 0.8)$ \\
\hline
\end{tabular}

OPNET настроен на сбор графической информации от различных сетевых устройств и источников данных, в том числе и видео трафика, маршрутизаторов, каналов и коммутаторов [4]. Моделирование каждого сценария длилось 900 секунд. Направление видео потока начинается на 70 секунде.

На рисунке 1, конвергенция сети с использованием EIGRP происходит быстрей, чем при использовании OSPF, так как при OSPF связность нарушается на 400 секунде и восстанавливается только на 800 секунде. Это обусловлено тем, что при обнаружении изменений в топологии сети, маршрутизатор, настроенный для работы с протоколом 
маршрутизации EIGRP, посылает запрос только к непосредственным соседям, для обнаружения successor, и распространения информации на все узлы.

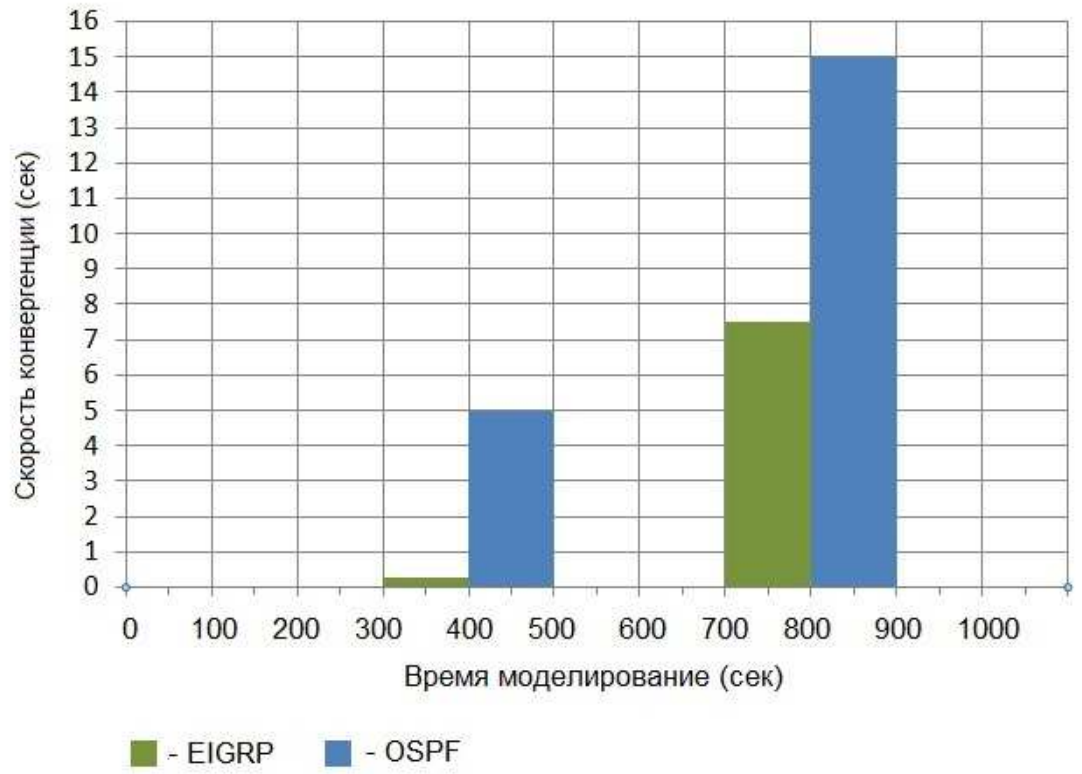

\section{Рисунок 1 - Отношение скорости конвергенции ко времени моделирования}

В случае использования OSPF, каналы сети переполняются LSA сообщениями, а так же, происходит перестроение таблиц маршрутизации. В связи с этим, OSPF затрачивает больше времени на конвергенцию.

Изменение задержки пакетов оказывает огромное влияние на потоковую передачу видео. На рисунке 2, показана задержка пакетов OSPF. Время задержки пакетов начинает расти приблизительно на 400-отой секунде моделирования и достигает примерно 1 секунды.

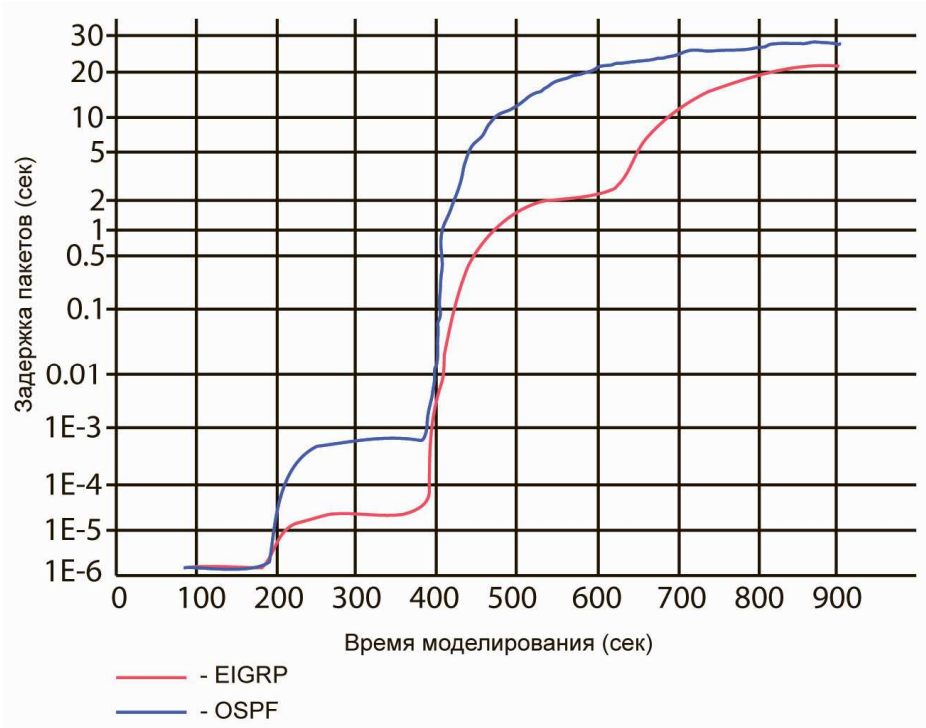

Рисунок 2 - Задержка получения пакетов 
В то же время, задержка пакетов на 400 секунде моделирования, при использовании EIGRP составляет около 1мс, а затем начинает резко возрастать. Нагрузка на сеть была установлена в несколько шагов, 20\%, 40\%, 60\% и $80 \%$ на 200, 400, 600 и 800 секундах соответственно. До 400 секунд, задержка в обоих случаях является меньше 30мс, что является приемлемым качеством передачи потокового видео в режиме реального времени. В случае EIGRP, задержка медленно возрастает после 400 секунд при сетевой нагрузке в 40\%. Так же, наблюдаются расхождения в задержке пакетов в интервале от 400 до 900 секунд. На рисунке 2 показано, что при изменении топологии сети, использование протокола маршрутизации EIGRP, приводит к меньшему значению задержки пакетов.

На рисунке 3 показаны задержки сквозного обслуживания. Как видно из графика, задержка при передаче пакетов в EIGRP и OSPF сетях, составляет около 70ms, начиная с 190 секунды до 395 секунды моделирования (при нагрузке на сеть в 20\%).

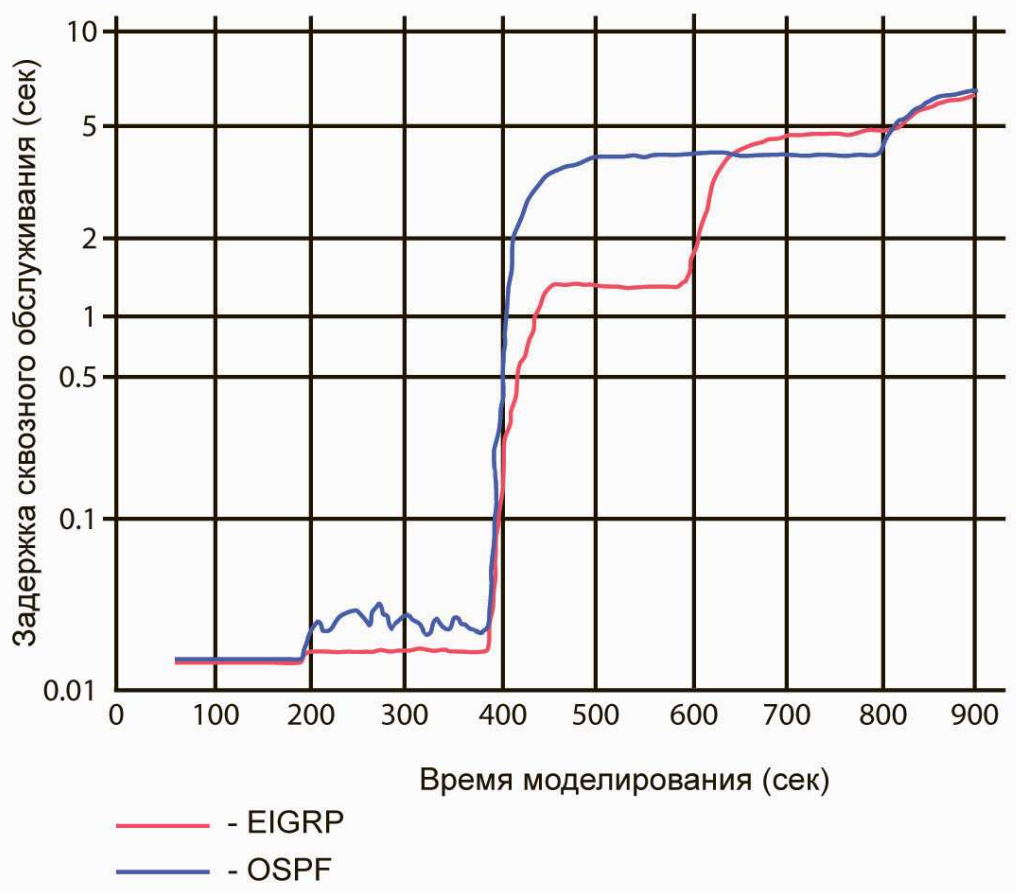

\section{Рисунок 3 - Задержки сквозного обслуживания}

Так же замечено, что при использовании EIGRP, задержка увеличивается до $200 \mathrm{~ms}$ на 400 секунде (при нагрузке в 40\%). При таких же условиях в сети OSPF, задержка составляет 1.5 секунды. Тем не менее, на 600 секунде моделирования, при повышении нагрузки на сеть до 60\%, показатели задержки, при использовании обоих протоколов маршрутизации, начинают возрастать, и постепенно увеличиваются до конца моделирования.

На рисунке 4 отображена пропускная способность сети при использовании рассматриваемых протоколов маршрутизации. 


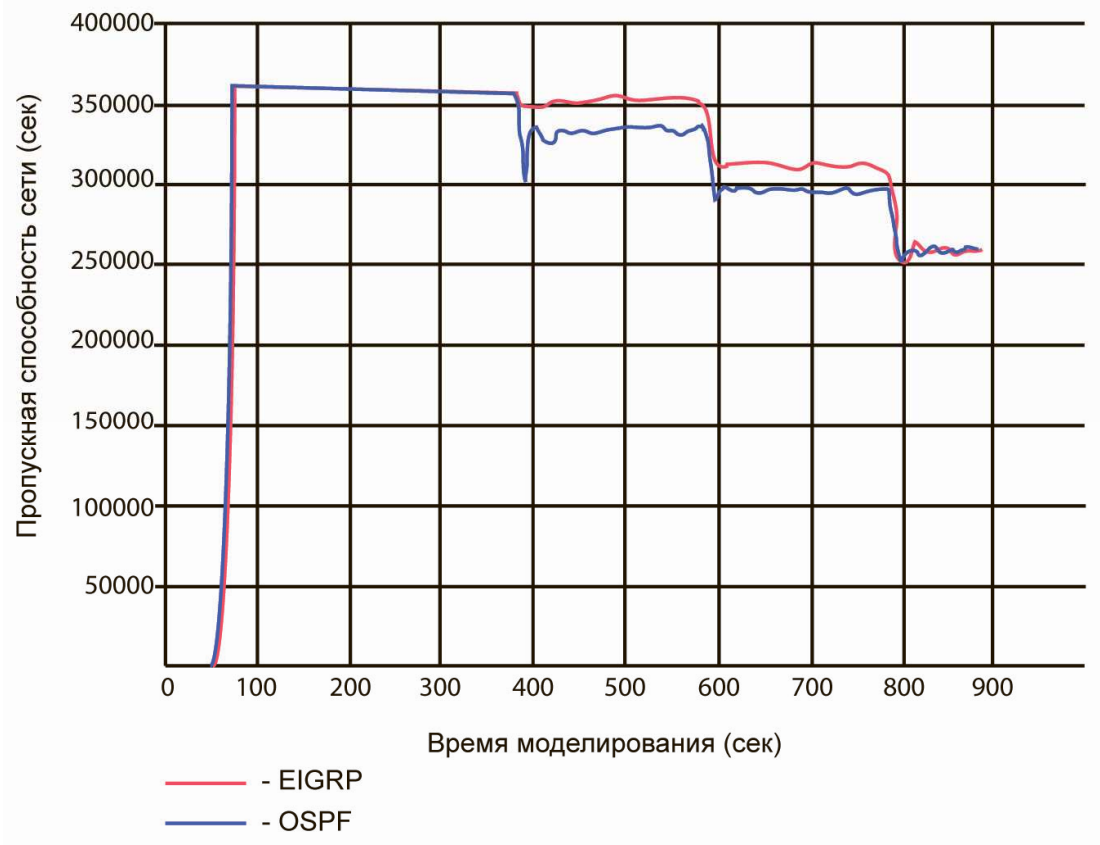

\section{Рисунок 4 - Пропускная способность сети}

Потеря пакетов наблюдается начиная с 400 секунды моделирования, как в сети с использованием EIGRP, так и в сети с использованием OSPF. График отображает воздействие на полученный трафик в зависимости от возрастания фоновых нагрузок. В сети с использованием EIGRP наблюдается потеря 0.14\% и 2.9\% передаваемых пакетов, при фоновых нагрузках 20\% и 40\% соответственно. Рассматривая OSPF, потери трафика составляют 0,35\% и 6\%, при одинаковых нагрузках. В момент нарастании фоновой нагрузки, количество потерянных пакетов возрастает, как при использовании EIGRP, так и OSPF. Исходя из результатов моделирования, можно сделать вывод, что сети, использующие в качестве протокола маршрутизации EIGRP, обладают большей пропускной способностью, по сравнению с сетями OSPF.

Моделирование для анализа было проведено в одной сети, с применением протоколов передачи данных используемых приложениями реального времени. Оценка эффективности протоколов маршрутизации была произведена на основе указанных критериев.

\section{Заключение}

Результаты моделирования позволяют утверждать, что производительность EIGRP лучше, по сравнению с OSPF.

Анализируя протоколы маршрутизации со стороны скорости сходимости, EIGRP быстрее восстанавливает связность сети, чем OSPF, благодаря более быстрому изучению топологии и обновлению таблиц маршрутизации. Показатели задержки пакетов, при изменении топологии сети, в сети с использованием OSPF, значительно выше, чем при использовании EIGRP. Таким образом, EIGRP обладает большей производительностью. Результаты моделирования так же показали, что сквозные задержки в сети EIGRP значительно меньше, чем в сети OSPF. Показатели сквозной 
задержки в сети EIGRP, на протяжении большего времени моделирования, находятся в допустимых пределах качества передачи потокового видео в режиме реального времени. Так же, в сети EIGRP, количество потерянных пакетов гораздо меньше, чем в сети OSPF, что свидетельствует о большей пропускной способности. EIGRP является гибридом дистанционно-векторных протоколов и протоколов состояния канала, благодаря этому, обеспечивает повышенную скорость конвергенции, минимальность задержек и хорошую пропускную способность.

\section{Список информационных источников}

[1] Nohl, A.R, Molnar G, "The convergence of the OSPF routing protocol" (Ericsson Res., Ericsson Hungary Ltd., Budapest, Hungary); Source: Periodica Polytechnica Electrical Engineering, v 47, n 1-2, p 89-100, 2002.

[2] "Understanding the protocols underlying dynamic routing". CNET Networks, Inc. Retrieved p.10-17, 2008.

[3] Олифер В.Г. Олифер Н.А. Компьютерные сети. Принципы, технологии, протоколы. Издательство, Питер, 4-е издание , 2010.

[4] Wendell Odom, CCNP ROUTE 642-902 Official Certification Guide, Cisco Press, 2010.

[5] Остроух А.В. Информационные технологии в научной и производственной деятельности / [ред. А.В. Остроух] - М: ООО "Техполиграфцентр", 2011. - 240 с. ISBN 978-5-94385-056-1.

[6] Саакян И.Э., Шушанский К.П. Учет и верификация мобильных пользователей корпоративных сетей // Автоматизация и управление в технических системах. 2012. - № 2. - C. 94-98. 